\title{
The interplay of StyR and IHF regulates substrate-dependent induction and carbon catabolite repression of styrene catabolism genes in Pseudomonas fluorescens ST Giordano Rampioni1,2, Livia Leoni ${ }^{1}$, Biancamaria Pietrangeli ${ }^{2}$ and Elisabetta Zennaro*1
}

Address: ${ }^{1}$ Department of Biology, University Roma Tre, Viale Marconi 446, 00146, Rome, Italy and ${ }^{2}$ Istituto Superiore per la Prevenzione e la Sicurezza sul Lavoro, Dipartimento Insediamenti Produttivi ed Interazioni con l'Ambiente, Via Urbana 167, 00184, Rome, Italy

Email: Giordano Rampioni - rampioni@uniroma3.it; Livia Leoni - leoni@bio.uniroma3.it;

Biancamaria Pietrangeli - biancamaria.pietrangeli@ispesl.it; Elisabetta Zennaro* - zennaro@uniroma3.it

* Corresponding author

Published: II June 2008

BMC Microbiology 2008, 8:92 doi:10.1 186/147/-2180-8-92
Received: 3I March 2008

Accepted: II June 2008

This article is available from: http://www.biomedcentral.com/I47I-2180/8/92

(C) 2008 Rampioni et al; licensee BioMed Central Ltd.

This is an Open Access article distributed under the terms of the Creative Commons Attribution License (http://creativecommons.org/licenses/by/2.0), which permits unrestricted use, distribution, and reproduction in any medium, provided the original work is properly cited.

\begin{abstract}
Background: In Pseudomonas fluorescens ST, the promoter of the styrene catabolic operon, PstyA, is induced by styrene and is subject to catabolite repression. PstyA regulation relies on the StyS/ StyR two-component system and on the IHF global regulator. The phosphorylated response regulator StyR (StyR-P) activates PstyA in inducing conditions when it binds to the high-affinity site STY2, located about -40 bp from the transcription start point. A cis-acting element upstream of STY2, named URE, contains a low-affinity StyR-P binding site (STYI), overlapping the IHF binding site. Deletion of the URE led to a decrease of promoter activity in inducing conditions and to a partial release of catabolite repression. This study was undertaken to assess the relative role played by IHF and StyR-P on the URE, and to clarify if PstyA catabolite repression could rely on the interplay of these regulators.

Results: StyR-P and IHF compete for binding to the URE region. PstyA full activity in inducing conditions is achieved when StyR-P and IHF bind to site STY2 and to the URE, respectively. Under catabolite repression conditions, StyR-P binds the STYI site, replacing IHF at the URE region. StyRP bound to both STYI and STY2 sites oligomerizes, likely promoting the formation of a DNA loop that closes the promoter in a repressed conformation. We found that StyR and IHF protein levels did not change in catabolite repression conditions, implying that PstyA repression is achieved through an increase in the StyR-P/StyR ratio.

Conclusion: We propose a model according to which the activity of the PstyA promoter is determined by conformational changes. An open conformation is operative in inducing conditions when StyR-P is bound to STY2 site and IHF to the URE. Under catabolite repression conditions StyR-P cellular levels would increase, displacing IHF from the URE and closing the promoter in a repressed conformation. The balance between the open and the closed promoter conformation would determine a fine modulation of the promoter activity. Since StyR and IHF protein levels do not vary in the different conditions, the key-factor regulating PstyA catabolite repression is likely the kinase activity of the StyR-cognate sensor protein StyS.
\end{abstract}




\section{Background}

Styrene is a basic building block for the manufacture of a broad range of products containing molecules such as polystyrene, butadiene-styrene latex, styrene copolymers and unsaturated polyester resins. These products range from packaging materials to food service items to a myriad of consumer electronics, construction, transportation and medical applications. Styrene exposure may cause contact-based skin inflammation, irritation of eyes, nose and respiratory tract, while neurological effects, such as alterations in vision, hearing loss and longer reaction times, have been associated with styrene exposure in the workplace [1]. Microbial biodegradation and polluted air biofiltration are attractive options for the removal of styrene from the environment, because they are cost-effective and do not generate secondary contaminants. Therefore styrene-degrading microorganisms have been receiving increasing interest, mainly concerning the factors that can help or impair the degradation process [2].

Despite the large number of bacteria isolated for their capability to grow on styrene, genetic studies have essen- tially been performed on strains belonging to the genus Pseudomonas [2]. In these strains styrene degradation starts with the oxidation of the vinyl double bond to styrene oxide by styrene monooxygenase (SMO), a two-component flavin-dependent oxygenase encoded by the sty $A$ and $s t y B$ genes, whose reaction mechanism has been proposed $[3,4]$. styC codes for styrene oxide isomerase (SOI), which converts styrene oxide to phenylacetaldehyde that is in turn oxidized to phenylacetic acid by phenylacetaldehyde dehydrogenase (PADH), encoded by styD [5-10]. The styE gene codes for a protein likely involved in the active transport of styrene [11]. These genes form an operon, named sty $A B C D E$, that is highly conserved in all the styrenedegrading Pseudomonas strains studied up to now (Figure 1A) [12]. Phenylacetic acid is a common substrate for Pseudomonas spp., probably because, beside styrene, degradation of many other aromatic compounds converge towards the formation of this compound [13].

In Pseudomonas fluorescens ST, as well as in the other styrene-degrading Pseudomonas strains, the expression of the styABCDE operon is regulated by the StyS/StyR two-com-
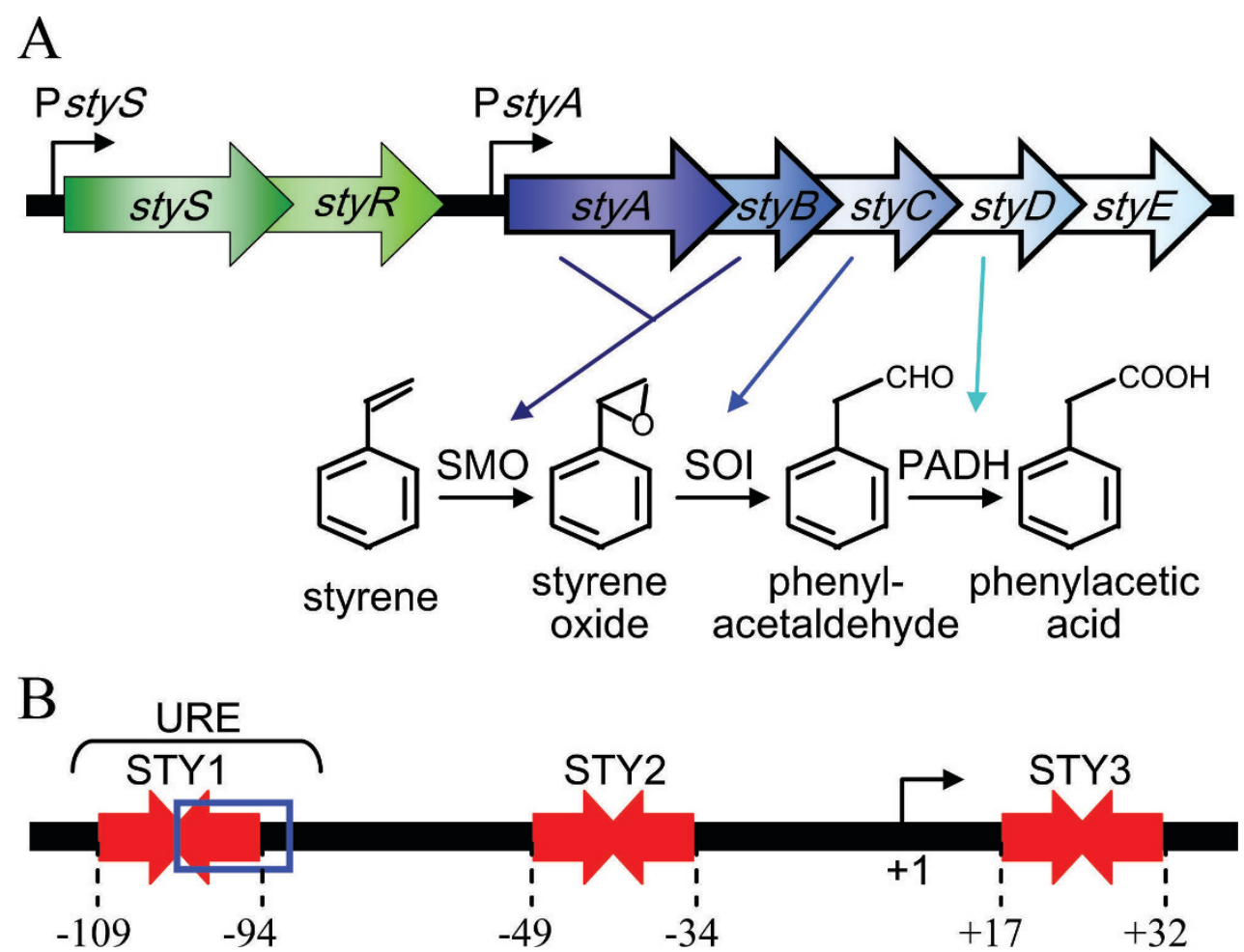

Figure I

The styrene-catabolic system of $\boldsymbol{P}$. fluorescens ST. (A) Organization of the stySR regulatory and of the styABCDE catabolic operons in Pseudomonas spp. styS, sensor histidine kinase; styR, response regulator; styA and styB, styrene monooxygenase (SMO); styC, styrene oxide isomerase (SOI); styD, phenylacetaldehyde dehydrogenase (PADH); styE, styrene transport protein. PstyS and PstyA are the promoters of the regulatory and catabolic operons, respectively. (B) Schematic representation of the PstyA promoter region. Inverted red arrows, StyR-P-binding sites STYI, STY2 and STY3 (numbering refers to distance from PstyA transcription start site). Blue box, IHF-binding site. Bent arrow, transcription start site. 
ponent system, encoded by the stySR operon (Figure 1A) $[9,10]$. The product of the styS gene, StyS, is a protein of about $109 \mathrm{kDa}$, predicted to be a hybrid histidine kinase (HK) with several distinct functional domains. A distinctive feature of this protein with respect to other hybrid HKs is the presence of two different kinase cores (HK/ ATPase domains). Moreover it contains an internal receiver domain and two putative input domains, consisting of the PAS and PAC sensory sub-domains [12]. The $s t y R$ gene encodes a protein, StyR, of about $23 \mathrm{kDa}$ disclosing the typical structural features of other response regulators [14]. Activation of StyR requires phosphorylation that triggers StyR dimerization and DNA binding [15].

Three distinct binding sites, endowed with different affinities for phosphorylated StyR (StyR-P), have been characterized on PstyA, the promoter of the styrene catabolic operon (Figure 1B) [16]. Site STY2 is the highest-affinity StyR-P binding site. The DNA region upstream of STY2, named URE (Upstream Regulatory Element), contains a lower affinity StyR-P binding site (STY1) overlapping a binding site for the Integration Host Factor (IHF) global regulator, placed in the opposite helix with respect to STY1 site. However, when a promoter fragment containing both STY1 and STY2 was used in DNase I protection experiments, we found that StyR-P bound these sites simultaneously, indicating that StyR-P binding to these sites is cooperative. The lowest affinity StyR-P binding site (STY3) is located downstream of the transcription start point [16].

PstyA activity is induced by styrene and partially repressed by the addition of glucose (60\% repression) or of other more favourable carbon sources [17]. This phenomenon, named carbon catabolite repression, is frequently observed in the regulation of aromatic catabolic pathways in pseudomonads, although, in most cases, little is known about the mediators or the mechanisms underlying repression. In their whole, the studies carried out up to date rule out in Pseudomonas the involvement of cyclic $\mathrm{AMP}$ and indicates that different genes are involved in the catabolite repression of different catabolic pathways [reviewed in $[18,19]]$.

Studies on the activity of deleted and/or mutated variants of PstyA, in cells grown in inducing (growth on styrene as sole carbon source) or catabolite repression (glucose added to a styrene growing culture) conditions, showed that StyR-P acts as an activator when it binds to site STY2, and that this binding is essential for promoter activity [20]. Conversely, deletion of the URE decreased PstyA activity and partially relieved it from glucose-mediated repression. When in this deleted promoter the STY3 site was also inactivated, glucose-mediated repression was completely abolished. From these data we concluded that
StyR-P acts as a repressor when it binds to site STY3, and that the URE region of PstyA is involved in both styrenedependent induction and catabolite repression [16]. However, the relative role played by IHF and StyR-P on this cis-acting element remained unclear.

This study was undertaken to address this issue and to understand if changes in the relative levels of StyR-P and IHF could determine PstyA catabolite repression.

The results obtained make it possible to propose a model according to which the fine regulation of the PstyA promoter, in the different growth conditions, mainly depends on the phosphorylation levels of StyR, thus on the activity of the cognate StyS sensor kinase.

\section{Results and discussion StyR-P and IHF compete for binding to the URE region}

The promoter region of the sty $A B C D E$ operon is shown in Figure 1B. The PstyA region located upstream of the STY2 site, referred to as URE (Upstream Regulatory Element), contains two cis-acting regulatory elements: an IHF binding site and the StyR-P binding site STY1 [16]. Since the STY1 and IHF binding sites overlap, it was postulated that StyR-P and IHF could compete for binding to the URE region. To clarify this issue, competition experiments were performed by DNase I protection assays on a DNA-probe encompassing site STY2 and the URE region of PstyA.

As shown in Figure 2, the IHF protection pattern (nucleotides -110/-75 from the PstyA transcription start site; Figure 2, lanes 7 and 15) is wider than the StyR-P protection pattern on STY1 (nucleotides -110/-90 from the transcription start site; Figure 2, lanes 1 and 9), so that they are easily distinguishable. The addition of increasing StyR-P concentrations (Figure 2, lanes 6 to 2) to the IHF/probe preformed complex, led to the protection of site STY2, and to the replacement of the IHF protection pattern with the narrower StyR-P protection pattern on the URE region. In Figure 2 triangles indicate the bands that are distinctive of the pattern obtained in the absence of IHF. Three of these bands become hypersensitive sites under increasing concentrations of StyR-P (indicated by open triangles in Figure 2), suggesting a conformational change of PstyA structure (see below). Likewise, the addition of increasing IHF concentrations (Figure 2, lanes 10 to 14 ) to the StyR$\mathrm{P} /$ probe preformed complex, led to the replacement of the StyR-P protection pattern at the STY1 site with the wider IHF protection pattern. In this case the binding of IHF to the URE region did not affect the binding of StyR-P to site STY2.

In the whole, these data demonstrate that IHF and StyR-P are in binding competition for the URE region, and that 


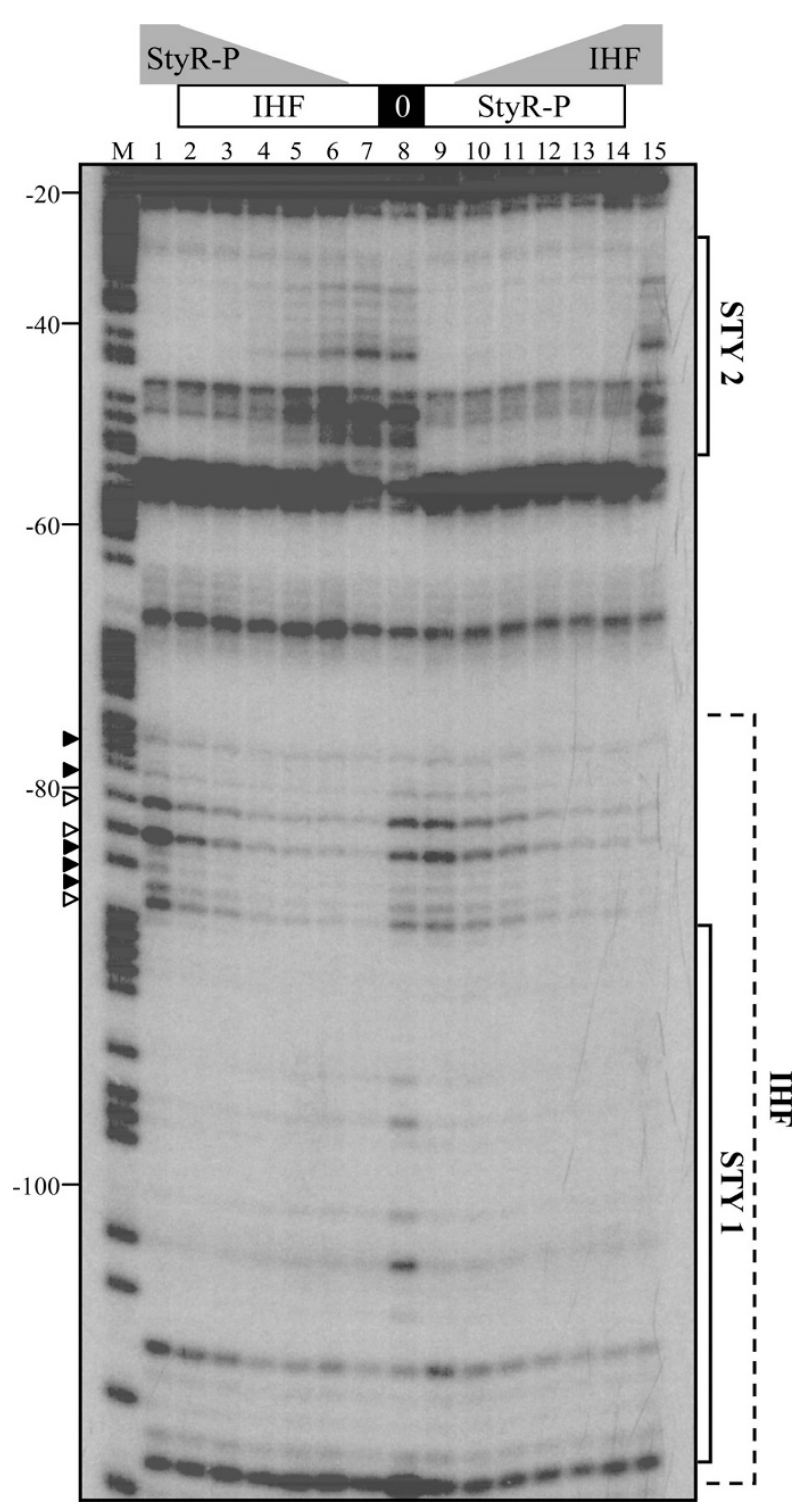

Figure 2

Characterization of StyR-P and IHF binding to the URE. DNase I protection assay in which a DNA fragment extending from - I45 to -8 (Top Strand) of the PstyA promoter was incubated with different amounts of IHF and/or StyR-P proteins prior to DNase I digestion. Numbering refers to PstyA transcription start site. Solid brackets indicate the regions showing specific protection by StyR-P; dashed bracket indicates the region showing specific protection by IHF. Triangles indicate bands that are distinctive of the protection pattern obtained in the absence of IHF (open triangles indicate hypersensitive sites). M, Maxam and Gilbert sequencing reactions $(A+G)$; IHF concentrations: lanes I, 8, and 9, no IHF added; lanes 2 to 7, $4.0 \mu \mathrm{M}$; lanes 10 to I5, 0.5 $\mu \mathrm{M}, \mathrm{I} .0 \mu \mathrm{M}, 2.0 \mu \mathrm{M}, 4.0 \mu \mathrm{M}, 8.0 \mu \mathrm{M}$ and $8.0 \mu \mathrm{M}$, respectively. StyR-P concentrations: lanes 7, 8, and I5, no StyR-P added; lanes I to 6, 8.0 $\mu \mathrm{M}, 8.0 \mu \mathrm{M}, 4.0 \mu \mathrm{M}, 2.0 \mu \mathrm{M}$, I.0 $\mu \mathrm{M}$ and $0.5 \mu \mathrm{M}$, respectively; lanes 9 to I4, $4.0 \mu \mathrm{M}$.
IHF can bind the URE region without altering StyR-P binding to site STY2.

\section{StyR-P and IHF play opposite roles when bound to the URE region}

In a previous work we showed that deletion of the URE led to a decreased promoter activity in inducing conditions (growth on styrene as sole carbon source) and to a partial desensitization to carbon catabolite repression (growth on styrene plus glucose). Actually, PstyA activity was $60 \%$ reduced in catabolite repression, while it was only $38 \%$ reduced in the URE-deleted variant of the promoter in the same growth conditions [16]. To better understand the contribution of StyR-P and IHF in determining the PstyA activity under styrene induction and glucose catabolite repression, we generated two variants of this promoter: Pa1STY1mut and Pa1IHFmut, each containing nucleotide substitutions such that impair StyR-P binding to site STY1 or IHF binding to the URE, respectively (Figure 3A). Electrophoretic mobility shift assays and DNase I protection assays were carried out to confirm that a DNA probe encompassing the URE region and carrying mutations inactivating STY1 (indicated with asterisks in Figure 3A) was able to form a complex with IHF, but not with StyR-P. As well, a DNA probe encompassing the URE and carrying mutations in the IHF binding site (indicated with triangles in Figure 3A) was able to form a complex with StyR-P, but not with IHF (data not shown).

Pa1STY1mut and Pa1IHFmut were cloned in the promoter-probe vector PPR9TT, in frame with the reporter gene lacZ, generating pPR9STY1mut and pPR9IHFmut (Table 1; Figure 3A), respectively, and introduced in P. fluorescens ST. We assayed the activity of these promoters with respect to that of the wild-type promoter $\mathrm{Pa1}$, monitoring $\beta$-galactosidase levels in inducing, repressing, and non-inducing conditions. Briefly, ST(pPR9Pa1), ST(pPR9STY1mut) and ST(pPR9IHFmut) strains pregrown on styrene, were diluted and subcultured in the same medium for two hours, and then divided into three flasks containing styrene (inducing condition), styrene plus glucose (repressing condition), and glucose alone (non-inducing condition) $[16,17]$. The $\beta$-galactosidase levels disclosed by the different constructs along the growth in the different culture conditions are shown in Figure $3 \mathrm{~B}$. The styrene pre-induced strains with the different constructs diluted the accumulated $\beta$-galactosidase at the same rate during the growth on glucose, a condition in which the PstyA promoter is not induced. This result made it possible to consider the $\beta$-galactosidase activity values obtained in this culture condition, for each strain and for each point of the growth curve, as $100 \%$ of repression of the PstyA promoter. Similarly, we assumed the $\beta$ galactosidase values observed along the exponential growth on styrene (inducing condition) as $100 \%$ of the 

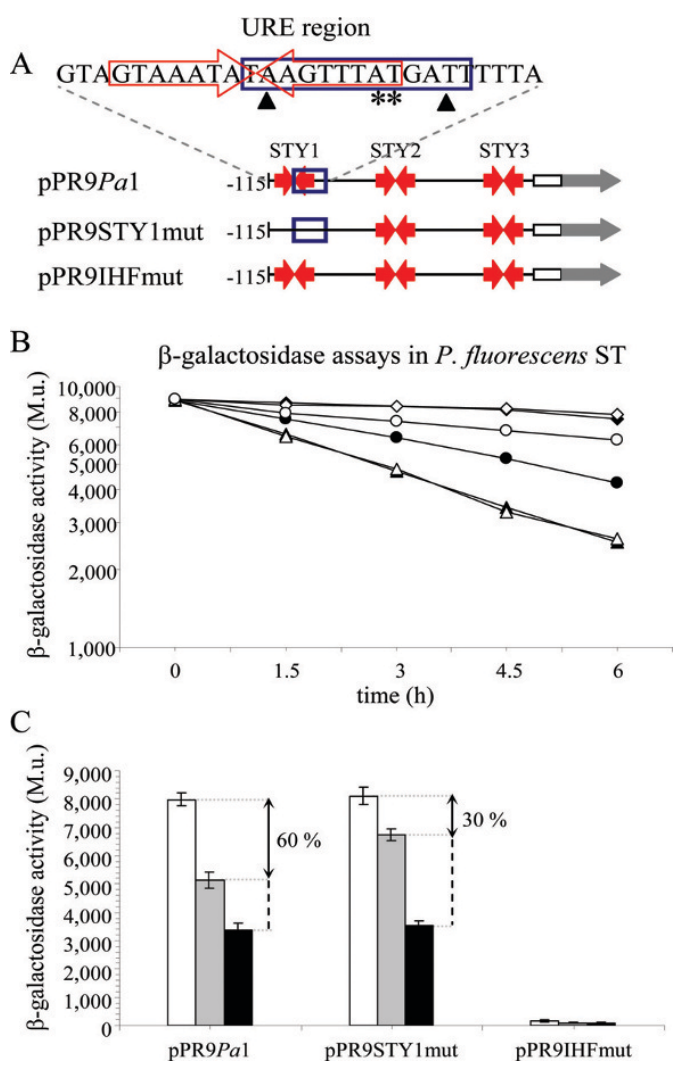

Figure 3

Functional role of the URE region. (A) Schematic representation of PstyA and its mutated derivatives cloned in the promoter probe vector pPR9TT. Plasmid designations are given on the left. Red inverted arrows indicate the StyR-P binding sites. The IHF binding site is blue-boxed. Nucleotides numbering is referred to the PstyA transcription start site. The white rectangle indicates the styA ORF. The grey arrow indicates the lac $Z$ gene fused to styA. A detail of the URE region is reported on the top. Asterisks and triangles indicate nucleotides mutated $(A \rightarrow T$ and $T \rightarrow A$ substitutions) to generate the PPR9STYImut and PPR9IHFmut constructs, respectively. (B) $\beta$-galactosidase activities disclosed by $P$. fluorescens $S T$ strains carrying the different $P$ PR9TT-derivative plasmids represented in (A). Filled symbols, pPR9Pal; open symbols, PPR9STY I mut; diamonds, cultures growing on styrene; circles, styrene-grown precultures to which both styrene and $0.4 \%$ glucose were added at time zero; triangles, styrene-grown precultures to which only $0.4 \%$ glucose was added at time zero. (C) $\beta$-galactosidase activities measured after two exponential cell divisions on styrene (white bars), on styrene plus $0.4 \%$ (wt/vol) glucose (grey bars), or on $0.4 \%$ (wt/vol) glucose (black bars), are reported in the histogram. The extent of glucose mediated repression in carbon catabolite repression condition is indicated by the double arrow line. Repression was calculated by assuming that the promoter activity in styrene was $100 \%$ and the promoter activity in glucose corresponded to $100 \%$ repression. Standard deviations (vertical lines) are based on the mean values of five independent experiments.
PstyA activity. Since the differences in promoter activity among the different strains kept constant during the growth curve, in Figure 3C a comparison of promoter activity after two exponential cell divisions, in the different culture conditions, is reported.

As shown in Figures 3B and 3C, mutation of the STY1 site in pPR9STY1mut produced no effect on the promoter activity under inducing conditions with respect to pPR9Pa1. Thus, this StyR-P-binding site is not involved in promoter activation. On the contrary, in the contemporary presence of styrene and glucose (repressing conditions), the same mutation caused a significant relief of repression (from 60\% repression disclosed by the wildtype promoter $\mathrm{Pa} 1$ to $30 \%$ repression) indicating that StyR-P bound to site STY1 plays a role in PstyA catabolite repression. We previously demonstrated that also site STY3 is responsible for a reduction of catabolite repression from $60 \%$ to $26 \%$ [16], so it seems that these lowaffinity StyR-P binding sites are both involved in PstyA repression.

As far as IHF is concerned, we observed no $\beta$-galactosidase activity in pPR9IHFmut in all the tested culture conditions. This was an unexpected result, since in a previous work we found that the deletion of the URE region led only to a $33 \%$ reduction of promoter activity in inducing condition with respect to pPR9Pa1 [16]. A possible interpretation of this finding is suggested by the fact that, in spite of the different affinity of StyR-P for the single STY sites, in the absence of IHF it binds STY1 and STY2 sites simultaneously [16]. This cooperative binding suggested that two dimers of StyR-P bound to sites STY1 and STY2 could interact, bending DNA and forming a repressive loop. An indication that major changes in the threedimensional structure of the promoter only occur when StyR-P is bound to STY1 and STY2 is the concomitant appearance of DNase I hypersensitive sites between these two sites (Figure 2, lane 1). These DNase I hypersensitive sites are not detectable when IHF displaces StyR-P from site STY1 and only STY2 is bound by StyR-P (Figure 2, lane 14). The formation of a StyR-P tetramer and DNA looping, probably favored by the presence of an intrinsically curved sequence between STY1 and STY2 [20], would impair RNA polymerase access to the promoter.

\section{StyR-P binding to sites STYI and STY2 promotes protein tetramerization}

In order to corroborate the hypothesis that the occupancy of both STY1 and STY2 sites by StyR-P induces the generation of a repressive DNA loop, we performed experiments aimed to assess if StyR-P dimers can form tetramers, and if this oligomerization is stimulated by the presence of a DNA fragment containing the StyR-P binding sites STY1 and STY2. 
Table I: Bacterial strains and plasmids used in this study.

\begin{tabular}{|c|c|c|}
\hline Strains and Plasmids & Relevant characteristics and plasmids contruction or source & Reference \\
\hline \multicolumn{3}{|l|}{ Strains } \\
\hline P. fluorescens ST & Sty ${ }^{+}$ & [39] \\
\hline E. coli $\mathrm{DH} 5 \alpha$ & 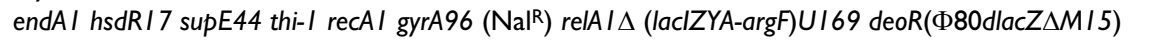 & Bethesda Res. Lab. \\
\hline \multicolumn{3}{|c|}{ (1) } \\
\hline pBluescript II KS+ & Cloning vector; $A p^{R} ; 2.9 \mathrm{~Kb}$. & Stratagene \\
\hline PPR9TT & lacZ promoter probe vector; $\mathrm{Ap}^{\mathrm{R}} ; \mathrm{Cm}^{\mathrm{R}}$. & {$[40]$} \\
\hline PTE50 & $\begin{array}{l}\text { PTZI9R derivative containing a } P \text {. fluorescens ST chromosomal fragment carrying the genes coding } \\
\text { for styR, sty } A \text { and styB; } A p^{R} \text {. }\end{array}$ & {$[7]$} \\
\hline pRK20I3 & Helper plasmid; ColEI replicon; Mob+; Tra+; Km². & [37] \\
\hline PSTYI/2 & $\begin{array}{l}\text { I } 55 \text { bp fragment, encompassing nucleotides }-145 \text { to }-8 \text {, with respect to the styA transcription start } \\
\text { point ligated to the EcoRI-Pstl sites of pBluescript II KS+; ApR. }\end{array}$ & {$[16]$} \\
\hline pPR9Pal & $\begin{array}{l}355 \text { bp fragment, encompassing nucleotides }-115 \text { to }+240 \text {, with respect to the styA transcription } \\
\text { start point, ligated to the Xhol-BamHI sites of pPR9TT; Ap } \text { P }^{\mathrm{C}} \mathrm{Cm}^{\mathrm{R}} \text {. }\end{array}$ & {$[20]$} \\
\hline pPR9STYImut & $\begin{array}{l}\text { Same construct as pPR9Pal, but the cloned sequence carries a double mutation in positions }-97 \text { and } \\
-96 \text { from the styA trascription start point; } \mathrm{Ap}^{\mathrm{R}} ; \mathrm{Cm}^{\mathrm{R}} \text {. }\end{array}$ & This study \\
\hline pPR9IHFmut & $\begin{array}{l}\text { Same construct as pPR9Pal, but the cloned sequence carries a double mutation in positions }-101 \\
\text { and }-9 \mid \text { from the styA trascription start point; } A p^{R} ; \mathrm{Cm}^{R} \text {. }\end{array}$ & This study \\
\hline
\end{tabular}

To determine the oligomerization state of StyR-P, the StyR protein was phosphorylated with acetylphosphate, and afterwards treated with the disuccinimidyl suberate (DSS) cross-linking agent, in absence or in the presence of increasing amounts of a DNA fragment encompassing the STY1 and STY2 sites. As shown in Figure 4A, StyR-P can form dimers of the predicted size of about $50 \mathrm{kDa}$ and higher order multimers. The size of the higher-order multimers of about $75 \mathrm{kDa}$ and $110 \mathrm{kDa}$ could be consistent with the formation of trimers and tetramers, respectively. However, native PAGE analysis performed on the acetylphosphate-treated sample showed that more than $99 \%$ of StyR was in the dimeric form, prior to the addition of DSS (data not shown) [12]. After cross-linking and denaturing SDS-PAGE analysis, more than $50 \%$ of the protein migrated as the monomeric form. This indicates that the extent of protein-protein cross-linking was far from complete, under these experimental conditions. Thus, it is likely that the band of about $75 \mathrm{kDa}$ derives from incomplete cross-linking of a tetramer rather than from the formation of a trimer.

The results of the cross-linking experiment highlight that StyR-P dimers are actually able to interact, forming a tetramer, also in the absence of DNA. Tetramers formation is significantly enhanced by addition of a DNA fragment encompassing sites STY1 and STY2 (Figure 4A), but not by the addition of non-specific DNA (Figure 4B). This specific DNA-mediated oligomerization is a strong indication that two StyR-P dimers bound on sites STY1 and STY2 form a StyR-P tetramer, likely inducing the formation of a DNA loop. Occurrence of changes in the promoter conformation when StyR-P is bound to both STY1 and STY2 is also indicated by the appearance of hypersensitive bands between these sites in DNase I protection experiments
(Figure 2). Moreover, a strong evidence of the formation of a "closed" promoter conformation that would impair RNA polymerase (RNAP) access to the promoter is the absence of PstyA activity in pPR9IHFmut. Taken together the above evidence concur to strengthen the significance of the cross-linking results.

\section{IHF has a positive role on PstyA activity by itself}

The above reported molecular and physiological results concur with the view that the cooperative binding of StyR$\mathrm{P}$ to STY1 and STY2 is likely counteracted in vivo by IHF which, competing with StyR-P for binding to the URE region, would act as a positive modulator of promoter activity. However, in a previous work we found that deletion of the entire URE led to a 33\% decrease in promoter activity in inducing conditions (growth on styrene as sole carbon source) with respect to the full length promoter [16]. From this result we argued that IHF could not merely have the role of displacing StyR-P from the repressive site STY1, since in this case no difference in promoter activity would have been found between the full length and the URE-deleted promoters. To clarify this issue, we compared the activity of pPR9Pa 1 and pPR9IHFmut in the heterologous host $E$. coli. As a control we also tested the $\beta$-galactosidase activity of pPR9STY1mut (Figure 5). In this system there was no StyR-P dependent activation of PstyA, but the promoter had a basal level activity anyway. This basal level activity was the same in pPR9Pa1 and in pPR9STY1mut, while it strongly decreased in pPR9IHFmut. This result demonstrates that IHF, beside displacing StyR-P from the STY1 repressive site, has also a positive effect on PstyA transcription by itself.

The molecular mechanism of action of IHF on the PstyA promoter remains an open issue. In many cases IHF bind- 


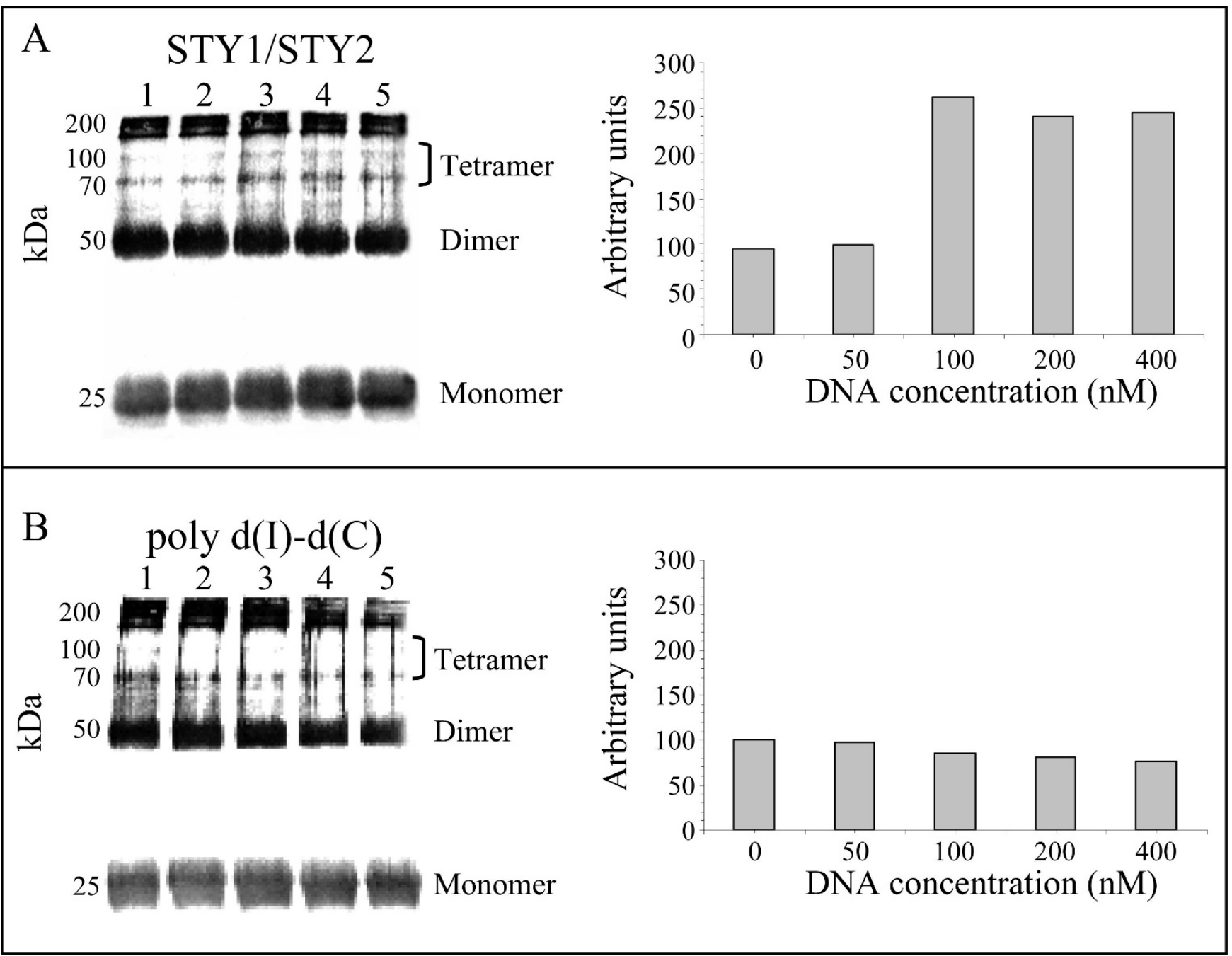

Figure 4

DNA-mediated StyR-P tetramerization. Western blot analysis of StyR-P (I00 nM) incubated with increasing amounts of the DNA fragment STYI/STY2 (panel A) or poly $\mathrm{d}(\mathrm{I})-\mathrm{d}(\mathrm{C})$ (panel B), and afterwards treated with the cross-linking agent DSS. Brackets indicate the $110 \mathrm{kDa}$ band corresponding to StyR-P tetrameric form, and the $75 \mathrm{kDa}$ band derived from incomplete cross-linking of tetramers (see text for details). Dimeric $(50 \mathrm{kDa})$ and monomeric $(25 \mathrm{kDa})$ StyR-P forms are indicated. The concentrations of DNA in each sample were: lane I, no DNA added; lanes 2 to 5, 50, 100, 200, 400 nM, respectively.

ing induces DNA-looping facilitating the interaction of an upstream regulatory element with RNAP [21]. However, our data rule out this possibility, since the IHF binding site is located at the very 5'- end of the minimum PstyA fragment endowed with full activity [20]. Therefore, it is likely that IHF acts as an activator through a different mechanism. For instance, it has been reported that IHF can activate transcription, without contacting RNAP, by facilitating duplex destabilization in the -10 region $[22,23]$.
PstyA catabolite repression does not rely on variation in StyR and IHF protein levels

Although the role of IHF in Pseudomonas physiology is far from understood, it has been often involved in the regulation of toxic compound degradation [18]. The above data show that binding of IHF to the URE exerts a positive effect on PstyA activity, under inducing conditions, while binding of StyR-P on the same region is involved in catabolite repression. Since StyR-P and IHF are in binding competition for the URE region, it is reasonable to suppose that, starting from an inducing condition (growth on styrene as sole carbon source), the glucose-mediated repression (growth on styrene and glucose) of PstyA could 


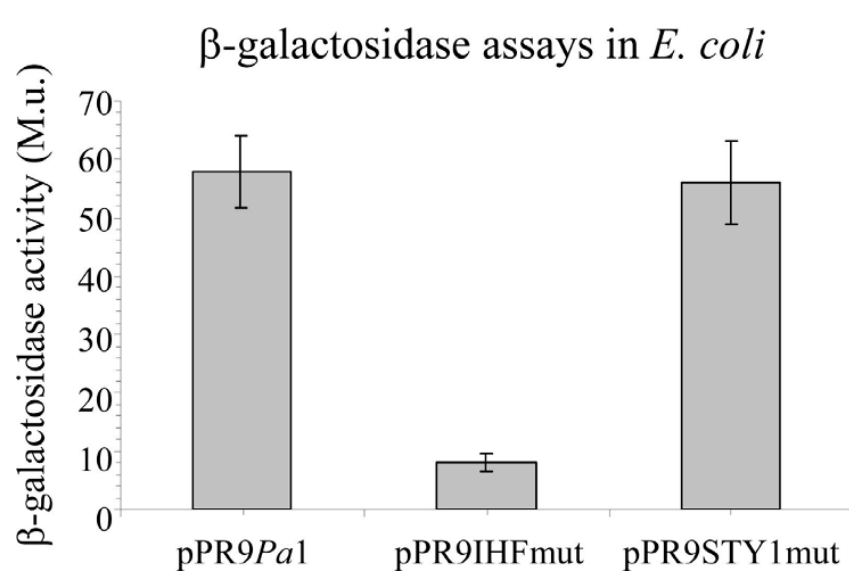

Figure 5

Role of IHF on PstyA activity. $\beta$-galactosidase activities disclosed by $E$. coli strains carrying the PPR9TT-derivative plasmids indicated below the histogram. The $\beta$-galactosidase assays were carried out on cells grown in LB medium at $37^{\circ} \mathrm{C}$ to an $A_{600} \cong 2.0$. Standard deviations (vertical lines) are based on the mean values of five independent experiments.

be due to an increase in StyR-P and/or to a decrease in IHF cellular levels.

To test this hypothesis, we monitored the levels of StyR and IHF proteins by western analysis after the addition of glucose to a styrene growing culture.

As previously described, P. fluorescens ST carrying pPR9Pa 1 plasmid was cultured with styrene as sole carbon source, then the culture was diluted, subcultured in the same medium for two hours, and divided into two flasks containing styrene, or styrene plus glucose. $\beta$-galactosidase levels were measured every 1.5 hours and protein samples for western analysis were withdrawn, in parallel, up to 7.5 hours after division. In inducing condition, StyR and IHF levels did not vary along the growth curve (data not shown), in accordance with PstyA steady levels (Figure 6A). Surprisingly, IHF levels did not vary and StyR levels did not increase, even when glucose was added to the styrene growing culture (Figure 6B), although PstyA activity decreased in this culture condition (Figure 6A).

This finding strongly suggests that in repressing conditions the low-affinity site STY1 is occupied as a consequence of an increase in the levels of phosphorylated StyR, and not for an increase in StyR or for a decrease in IHF absolute protein levels.

\section{Conclusion}

In our previous studies we showed that StyR dimerizes upon phosphorylation and that StyR-P dimers are able to bind PstyA on three distinct cis-acting elements (STY1,

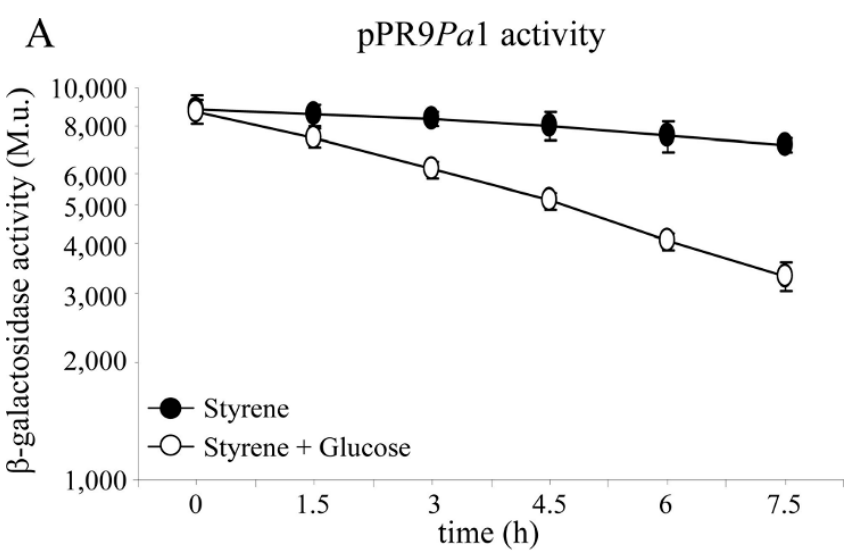

B

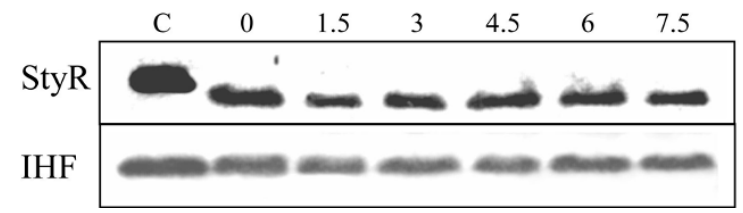

Figure 6

Analysis of StyR and IHF protein levels. (A) $\beta$-galactosidase assays performed with $P$. fluorescens ST strains carrying the pPR9Pal plasmid (Table I), grown in styrene (filled circles) and styrene plus $0.4 \%$ (wt/vol) glucose (open circles), along the growth curve. (B) Western hybridization performed with anti-StyR and anti-IHF antibodies on the cellular soluble fractions derived from the styrene plus glucose culture. Sampling times are indicated above the bands, and correspond to the sampling times of the $\beta$-galactosidase assays shown in (A); lane C, control with purified StyR and IHF proteins.

STY2 and STY3) with different affinity $[15,16]$. Functional studies showed that the high-affinity binding site STY2 is essential for promoter activation, while the low-affinity site STY3 is in part responsible for catabolite repression of the promoter. The URE region, encompassing the StyR-P binding site STY1 and the IHF binding site, is involved in both styrene-induction and catabolite repression of PstyA [16].

In this study we unravel the role of the URE region in PstyA regulation, showing that the mutually exclusive binding of StyR-P and IHF to this cis-acting element causes opposite effects on PstyA activity. Moreover, we demonstrate that the levels of IHF and StyR do not change in the different growth conditions, making the phosphorylation degree of StyR the main factor in the regulation of styrene catabolism genes expression.

On the whole, our research allows us to propose a model for PstyA regulation according to which the PstyA activity is determined by changes of its structure. An open conformation is operative in inducing conditions when StyR-P is 
bound to STY2 site and IHF to the URE. Under catabolite repression conditions StyR-P cellular levels would increase, displacing IHF from the URE and resulting in the achievement of a promoter closed conformation. Also the occupation of STY3 site would take part to the closed promoter conformation, since we had previously demonstrated that its inactivation partially relieved PstyA from glucose repression (Figure 7) [16]. The balance between

\section{A Open Conformation}

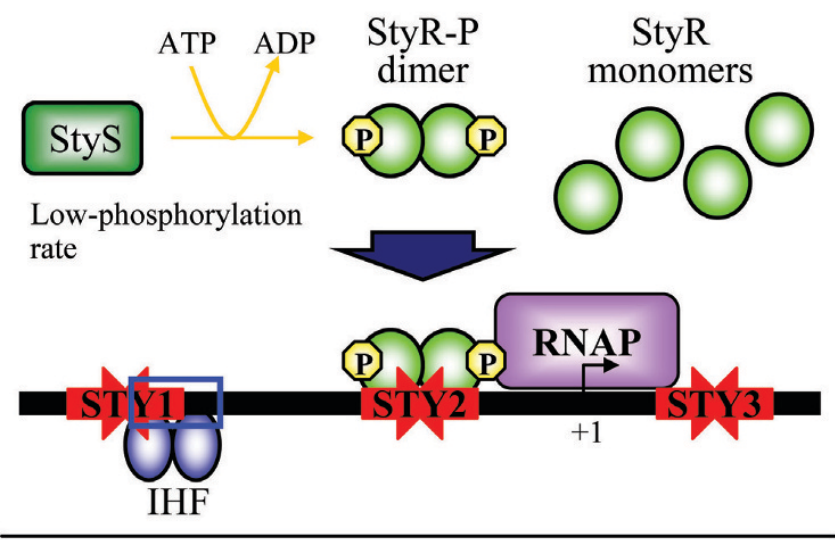

B Closed Conformation

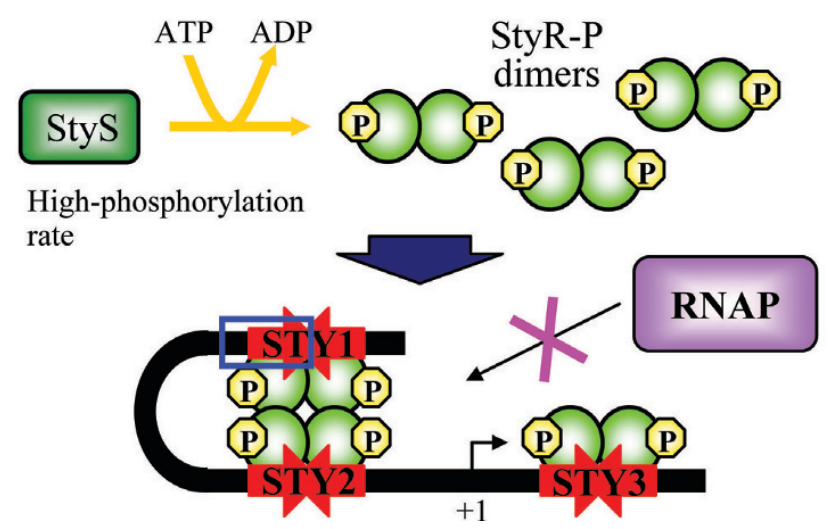

Figure 7

Proposed model for the regulation of PstyA. (A) When growing on styrene as sole carbon source, the StyS kinase activity leads to levels of StyR-P such that this protein binds only to the high-affinity actvating-site STY2, while IHF binds to the URE region. This "open" conformation would allow the binding of the RNA polymerase (RNAP) to PstyA, promoting transcription. (B) In carbon catabolite repression conditions (growth on styrene plus glucose) the increased StyS kinase activity would lead to higher levels of StyR-P, so that this protein can bind also to the low-affinity repressivesites STYI and STY3, displacing IHF from the URE region. This "closed" promoter conformation would impair the binding of the RNAP to the promoter. The balance between open and closed promoter conformations would determine a fine modulation of the promoter activity. the open and the closed promoter conformations would determine a fine modulation of PstyA activity.

In this perspective the key-factor regulating carbon catabolite repression would be the activity of the StyR-cognate sensor protein StyS. Our data converge to the notion that the activity of the sensor kinase StyS is enhanced when, besides styrene, cells are in a condition that determines a high redox potential (such as growth on styrene plus glucose). This view is consistent with the presence of two PAS domains and two kinase domains in the StyS sensor, which is a strong indication that two different signals can be sensed by this protein. If the two signals are styrene and the cell redox potential, a signal often perceived by PAS domains, their integration could modulate the kinase activity of the sensor, leading to an increase in the levels of phosphorylated StyR, under carbon catabolite repression conditions, and consequently to PstyA down-regulation [24].

Concerning the physiological role of IHF, in E. coli this protein regulates more than 100 genes and in Pseudomonas it is involved in the regulation of several processes, especially in toxic compound degradation $[18,25]$. Previous studies showed that in E. coli and Pseudomonas IHF levels increase upon entry into stationary phase in LB growing cultures and suggested a role for IHF in stress response $[26,27]$. It is likely that in $P$. fluorescens ST we did not observe the same phenomenon because we used minimal medium or because styrene is a stress factor by itself.

The TodS/T regulatory system of toluene catabolism in $P$. putida strain DOT-T1E strongly resembles the StyS/R system. The TodT response regulator binds with different affinity three sites of the promoter PtodX and IHF is essential for activating transcription $[28,29]$. However, in this system all these TodT binding sites have a positive effect on promoter activation and the role of IHF is to bend DNA, hence favouring the contact between the TodT activator bound further upstream and the $\alpha$-subunit of RNA polymerase. Accordingly, the position of TodT and IHF binding sites on PtodX promoter is very different from that of StyR and IHF binding sites on PstyA. The divergent evolution of StyS/R-PstyA and TodS/T-PtodX probably reflects the need for integrating these peripheral pathways into the global regulatory circuits of the corresponding different strains.

In Pseudomonas the majority of the catabolic routes for toxic compounds are regulated by a specific regulator responding to the actual substrate and by one or more global regulators devoted to couple the activity of the catabolic operon promoter to cell metabolism $[18,19,30]$. With this respect, the styrene-catabolism regulatory system is peculiar because a unique regulatory device (the 
StyS/StyR two component system) integrates the response to the specific stimulus (styrene) with the utilization of a higher-energy carbon source.

The fine modulation exerted by the interplay of StyR-P and IHF on PstyA activity, although complex, could not be the only regulatory device controlling styrene catabolism in P. fluorescens ST. It was recently reported that in Pseudomonas sp. Y2, in which the sty regulatory and catabolic genes are highly homologous to those of strain ST, the repressor PaaX represses PstyA in the absence of phenylacetyl-CoA, the first intermediate metabolite of the styrene degradation lower-pathway [31]. The authors suggested that PaaX could have a role in coordinating the expression of the upper and lower styrene catabolic pathways to avoid the accumulation of toxic catabolic intermediates such as styrene oxide and phenylacetaldehyde. We believe that both StyR-P and PaaX repressive mechanisms can be operative also in ST strain, probably acting in different metabolic conditions.

In the experimental design here reported we found that when styrene-induced cells are transferred to a medium containing both styrene and glucose, both carbon sources are simultaneously utilized (since PstyA activity is diminished, but not abrogated). Conversely, when non-induced cells are transferred to a medium containing both glucose and styrene, they exhibit a typical diauxic growth, in which glucose is utilized first [17]. Therefore it seems that catabolite repression could be achieved through different mechanisms, depending on the metabolic status of the cells preceding the addition of the alternative carbon source. It could be that when styrene is added to a glucose growing culture, the available energy is invested in adaptation to the high-toxic styrene, prior to metabolizing it. Conversely, when cells are already adapted to styrene and are productively using it as carbon source, there is not much advantage to completely repress styrene degradation in favor of glucose utilization, and the two carbon sources are thus exploited in parallel. The simultaneous utilization of an high-energy carbon source and of an aromatic compound has also been reported by J.L. Ramos and co-workers, who demonstrated that in $P$. putida KT2440 (pWWO) cells utilize glucose and toluene simultaneously [32].

In addition to its relevance in understanding the physiological mechanisms underlying aromatic compound catabolism, this study could lay the basis for the generation of engineered strains desensitized to carbon catabolite repression, thus improved in styrene catabolic potential.

\section{Methods}

\section{Bacterial strains, plasmids, media and chemicals}

The bacterial strains and plasmids used in this study are listed in Table 1. P. fluorescens ST and E. coli cells were routinely grown at $30^{\circ} \mathrm{C}$ and $37^{\circ} \mathrm{C}$, respectively, in Luria-Bertani (LB) medium or mineral salts medium supplemented with styrene, or $0.4 \%(\mathrm{wt} / \mathrm{vol})$ glucose, or both $[33,34]$. Styrene was added via the gas phase as previously described [7]. When necessary, cultures were supplemented with ampicillin (Ap, $100 \mu \mathrm{g} / \mathrm{ml}$ ), kanamycin $(\mathrm{Km}, 50 \mu \mathrm{g} / \mathrm{ml})$ or chloramphenicol $(\mathrm{Cm}, 30 \mu \mathrm{g} / \mathrm{ml}$ for $E$. coli; $200 \mu \mathrm{g} / \mathrm{ml}$ for $P$. fluorescens ST).

\section{Recombinant DNA techniques}

Details on the construction of plasmids are described in Table 1. Preparation of plasmid DNA, purification of DNA fragments, restrictions, ligations and transformations of E. coli, were carried out by standard procedures $[15,33]$. PCR amplifications were performed using Pfu polymerase (Stratagene) and the pTE50 plasmid as DNA template (Table 1) [7]. The sequences of oligonucleotides used in this study are shown in Table 2. Automated sequences were performed by MWG Biotech sequence services (MWG Biotech).

Table 2: Oligonucleotides used in this study.

\begin{tabular}{|c|c|c|c|}
\hline Name & Sequence $\left(5^{\prime}-3^{\prime}\right)^{a}$ & Positionb & Site $^{c}$ \\
\hline $\mathrm{PI} / \mathrm{FW}$ & GCTCTAGAGGTGTAGTAAATATAAGT & -115 & Xbal \\
\hline PI69/FW & GCTCTAGAGGTGTAGTAAATATTAGT & -115 & Xbal \\
\hline P87/FW & TAAATATAAGTAAATGATTTTTAAT & -108 & - \\
\hline P89/FW & TAAATATIAGTTTATGA_ATTTTAAT & -108 & - \\
\hline P86/RV & 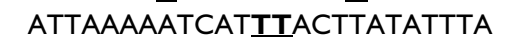 & -84 & - \\
\hline $\mathrm{P} 88 / \mathrm{RV}$ & ATTAAAAITCATAAACTAATATTTA & -84 & - \\
\hline PI7/RV & GGGGTACCTACGTAGTAGTAGTGG & +274 & Kpnl \\
\hline P35/FW & GGGAATTCCGTTGACTGCTTCGGG & -145 & EcoRI \\
\hline P56/RV & AAACTGCAGAGCTAACACCAGCAGC & -8 & Pstl \\
\hline
\end{tabular}

a introduced restriction sites are underlined; the nucleotides in bold-face and underlined correspond to the $\mathrm{A} \rightarrow \mathrm{T}$ and $\mathrm{T} \rightarrow \mathrm{A}$ introduced substitutions with respect to the styA wild-type sequence.

b distance in bp from PstyA transcription start point [10].

c restriction recognition sites. 


\section{DNase I protection assay}

The DNase I protection assay was performed as previously described [16]. In brief, the probe derived from EcoRI/SacI digestion of the pSTY1/2 plasmid (Table 1) and encompassing nucleotides $-145 /-8$ with respect to PstyA transcription start point was labelled by fill-in with $[\alpha-32 \mathrm{P}]$ dATP [16]. The labelled probe ( $0.5 \mathrm{nM}$ concentration) was mixed with different amounts of phosphorylated StyR (StyR-P) (0.5 to $8.0 \mu \mathrm{M}$, as indicated in legend of Figure 2) and/or IHF (0.5 to $8.0 \mu \mathrm{M}$, as indicated in legend of Figure 2) in "Phosphorylation Buffer" (43 mM Tris-acetate $\mathrm{pH} 8.0,30 \mathrm{mM}$ potassium acetate, $8 \mathrm{mM} \mathrm{MgCl}_{2}, 27$ $\mathrm{mM}$ ammonium acetate, $1 \mathrm{mM}$ DTT, $80 \mathrm{mM} \mathrm{KCl}, 10 \%$ (vol/vol) glycerol, 4\% (wt/vol) polyethylene glycol, 100 $\mu \mathrm{g} / \mathrm{ml}$ bovine serum albumin) containing $0.1 \mu \mathrm{g} / \mu \mathrm{l}$ poly(dI-dC) and $2 \mathrm{mM} \mathrm{CaCl}_{2}$. In vitro phosphorylation of StyR was previously described [15]. DNA-protein complexes were allowed to form at $30^{\circ} \mathrm{C}$ for $15 \mathrm{~min}$ in a total volume of $50 \mu \mathrm{l}$ for reaction. After $1 \mathrm{~min}$ at $25^{\circ} \mathrm{C}$, DNase I $(0.4 \mathrm{u}$; Roche Biochemicals $)$ was added to the reaction mixtures. The reaction mixture was incubated for $1 \mathrm{~min}$ at $25^{\circ} \mathrm{C}$ and then stopped by the addition of $150 \mu \mathrm{l}$ of "Stop Solution" (0.2 M sodium acetate $\mathrm{pH} 7.0,0.1 \mathrm{M}$ ethylenediaminetetraacetic acid pH 8.0, 0.15\% (wt/vol) sodium dodecyl sulfate, $100 \mu \mathrm{g} / \mathrm{ml}$ tRNA). DNA from the footprinting mixture was phenol-chloroform extracted, ethanol-precipitated and dissolved in $5 \mu \mathrm{l}$ of "Sequence Loading Buffer" [33]. After 3 min denaturation at $95^{\circ} \mathrm{C}$, DNA was loaded on a 7\% DNA sequencing gel [33]. The A+G Maxam and Gilbert reaction was carried out with the same probe and loaded on the gel along with the footprinting samples [33].

\section{Construction of PstyA::lacZ fusions and $\beta$-galactosidase assays}

The construction of pPR9TT-derivatives pPR9Pa1 has been previously described [20]. For the construction of plasmids pPR9STY1mut and pPR9IHFmut, a site-directed mutagenesis of PstyA was performed by the "Splicing by Overlap Extension PCR" method using the primers described in Table 2, as previously described $[35,36]$. These oligonucleotides are mutually complementary and correspond to a DNA region located from nt -108 to -84 with respect to the styA transcription start point. The $\mathrm{A} \rightarrow$ $\mathrm{T}$ and $\mathrm{T} \rightarrow \mathrm{A}$ introduced substitutions, with respect to the wild-type sequence, are indicated in Table 2 . In the first step, two distinct PCR reactions (PCR-1 and PCR-2) were carried out using chromosomal plasmid pTE50 (Table 1) as template to introduce the mutation in the PCR products. In the PCR-1 Pa1STY1mut was amplified with primers $\mathrm{P} 1 / \mathrm{FW}$ and P86/RV, while Pa1IHFmut with primers $\mathrm{P} 169 / \mathrm{FW}$ and P88/RV. In the PCR-2 reactions, the reverse primer was $\mathrm{P} 17 / \mathrm{RV}$, while the forward primer was $\mathrm{P} 87 /$ FW for Pa1STY1mut and P89/FW for Pa1IHFmut. In the second PCR step the products from PCR-1 and PCR-2 were used as both primers and templates. In this step a total DNA amount (PCR-1 + PCR-2) of $125 \mathrm{ng}$ has been used in a $100 \mu \mathrm{l}$ reaction. After the first 5 cycles $\left(94^{\circ} \mathrm{C}, 1\right.$ $\left.\min ; 68^{\circ} \mathrm{C}, 1 \mathrm{~min} ; 72^{\circ} \mathrm{C}, 1 \mathrm{~min}\right)$, the reverse primer P17/ RV and either primer: P1/FW or P169/FW, were added and the reaction was continued for 25 cycles.

The different PCR products were first blunt-cloned in HincII-digested pBluescriptII KS+ (Stratagene) and checked by sequencing. Afterwards, the fragments cloned in the right orientation were excised by XhoI-BamHI digestion and ligated to compatible sites of the promoter probe vector pPROTT (Table 1 ) in frame with the lacZ reporter gene. All pPR9TT-derivatives were transferred from E. coli to P. fluorescens ST by triparental matings with the helper plasmid pRK2013 (Table 1) [37].

In order to measure $\beta$-galactosidase activity, $P$. fluorescens ST cells harboring pPR9TT-derived plasmids, were grown for $12 \mathrm{~h}$ at $30^{\circ} \mathrm{C}$ in mineral salts medium (MM) supplemented with styrene as carbon source; cells were then diluted to a cell density corresponding to $A_{600} \cong 0.05$ in the same medium and subcultured until cultures reached $\mathrm{A}_{600} \cong 0.2$. Cultures were then divided into three flasks and $0.4 \%$ (wt/vol) glucose was added to one flask. Samples were withdrawn, every 1.5 hours, during the entire growth curve and $\beta$-galactosidase activity was measured as described by Miller [38]. The $\beta$-galactosidase assays performed in the E. coli strains carrying the different pPR9TTderivative plasmids were carried out on cells grown in LB medium at $37^{\circ} \mathrm{C}$ to an $\mathrm{A}_{600} \cong 2.0$.

\section{Protein-protein cross-linking assay}

The DNA fragment STY1/STY2 (nucleotides -145 to -8 with respect to PstyA transcription start point) used to promote StyR-P tetramerization was obtained by PCR amplification with the primers P35/FW and P56/RV described in Table 2. StyR-P (100 nM) was incubated with different concentrations of the STY1/STY2 PCR product or in presence of poly d(I)-d(C) (from 50 to $400 \mathrm{nM}$ ) in "Binding Buffer" (20 mM Tris-HCl, 2 mM EDTA, $5 \mathrm{mM} \mathrm{MgCl}_{2}, 30$ $\mathrm{mM} \mathrm{KCl}, 5 \%$ (vol/vol) glycerol, $0.025 \%$ (vol/vol) Nonidet P-40, and $30 \mu \mathrm{g} / \mathrm{ml}$ poly (dI-dC); pH 8.0). After 15 min of incubation at $30^{\circ} \mathrm{C}$, disuccinimidyl suberate (DSS) was added to a final concentration of $15 \mathrm{mM}$, followed by incubation at room temperature for $60 \mathrm{~min}$. To stop the cross-linking reaction, $5 \mu$ l of "6X Protein Sample Buffer" was added to the samples [33]. Cross-linking products were separated by electrophoresis on a $10 \%$ (wt/ vol) sodium dodecyl sulfate-polyacrylamide gel [33]. Proteins were electroblotted to nitrocellulose and StyR was detected with murine anti-6xHis monoclonal antibodies (Qiagen) and alkaline phosphatase-conjugate goat antimouse secondary antibody [15]. 


\section{Polyclonal antiserum production and Western analyses} Purified StyR $(200 \mu \mathrm{g})$ was emulsioned with complete Freund's adjuvant (Sigma) and used to immunize a mouse by intramuscular injection. After 3 weeks, a second boost of StyR $(150 \mu \mathrm{g})$ in complete Freund's adjuvant (Sigma) was given, followed by a third boost $(100 \mu \mathrm{g})$ at the sixth week. The animal was bled 2 weeks later and the serum was stored at $4{ }^{\circ} \mathrm{C}$. Animal experiments were performed according to the legislative decree $116 / 92$ by the Italian Ministry of Health. Western blotting and immunohybridization were carried out with anti-StyR antiserum $(1: 1,500)$, anti-IHF antiserum $(1: 1,000)$ and alkaline phosphatase-conjugate anti-mouse IgG as secondary antibody (1:5,000; Promega) [33]. Final development was performed with the "BCIP/NBT Color Development Substrate" (Promega), as recommended by the manifacturer. Anti-IHF antiserum was a generous gift from Prof. S.D. Goodman, University of Southern California, Los Angeles, CA, USA.

\section{Authors' contributions}

GR and LL performed research, participated in the design of the study and helped to write the manuscript. BP participated in the design of the study. EZ conceived of the study, participated in its design and coordination, and wrote the manuscript. All authors read and approved the final manuscript.

\section{Acknowledgements}

We express our gratitude to V. de Lorenzo (Centro Nacional de Biotecnologia, Madrid, Spain) and to S.D. Goodman (University of Southern California, Los Angeles, CA, USA) for their generous donation of IHF protein and anti-IHF polyclonal antibodies. We are also very obliged to $\mathrm{V}$. Pallottini (University Roma Tre, Rome, Italy) for the production of polyclonal antibodies anti-StyR. GR was supported by a grant from ISPESL (Istituto Superiore per la Prevenzione e la Sicurezza sul Lavoro). This work was supported by a grant from University Roma Tre (CLAR 2007).

\section{References}

I. U.S. Environmental Protection Agency, Integrated Risk Information System, Styrene [http://www.epa.gov/iris/subst/ 0104.htm]

2. O'Leary ND, O'Connor KE, Dobson ADW: Biochemistry, genetics and physiology of microbial styrene degradation. FEMS Microbiol Rev 2002, 26:403-4I7.

3. Otto K, Hofstetter K, Röthlisberger M, Witholt B, Schmid A: Biochemical characterization of StyAB from Pseudomonas sp. Strain VLBI20 as a two-component flavin-diffusible monooxygenase. J Bacteriol 2004, I 86:5292-5302.

4. Kantz A, Chin F, Nallamothu N, Nguyen T, Gassner GT: Mechanism of flavin transfer and oxygen activation by the two-component flavoenzyme styrene monooxygenase. Arch Biochem Biophys 2005, 442:102-116.

5. Utkin IB, Yakimov MM, Matveeva LN, Kozlyak El, Rogozhin IS, Solomon ZG, Bezborodov AM: Degradation of styrene and ethylbenzene by Pseudomonas species Y2. FEMS Bicrobiol Lett 1991, 77:237-242.

6. O'Connor K, Buckley CM, Hartmans S, Dobson ADW: Possible regulatory role for nonaromatic carbon sources in styrene degradation by Pseudomonas putida CA-3. Appl Environ Microbiol 1995, $61: 544-548$.

7. Marconi AM, Beltrametti F, Bestetti G, Solinas F, Ruzzi M, Galli E, Zennaro $\mathrm{E}$ : Cloning and characterization of styrene catabo- lism genes from Pseudomonas fluorescens ST. Appl Environ Microbiol 1996, 62:121-127.

8. Beltrametti F, Marconi AM, Bestetti G, Colombo C, Galli E, Ruzzi M, Zennaro E: Sequencing and functional analysis of styrene catabolism genes from Pseudomonas fluorescens ST. Appl Environ Microbiol 1997, 63:2232-2239.

9. Panke S, Witholt B, Schmid A, Wubbolts MG: Towards a biocatalyst for (S)-styrene oxide production: characterization of the styrene degradation pathway of Pseudomonas sp. Strain VLB I 20. Appl Environ Microbiol 1998, 64:2032-2043.

10. Velasco A, Alonso S, Garcia JL, Perera J, Diaz E: Genetic and fuctional analysis of the styrene catabolic cluster of Pseudomonas sp. Strain Y2. J Bacteriol 1998, 180:1063-1071.

II. Mooney A, O'Leary ND, Dobson ADW: Cloning and functional characterization of the styE gene, involved in styrene transport in Pseudomonas putida CA-3. Appl Environ Microbiol 2006, 72: $1302-1309$.

12. Leoni L, Rampioni G, Zennaro E: Styrene, an unpalatable substrate with complex regulatory networks. In Pseudomonas, a model system in biology Volume 5. Edited by: Ramos JL, Filloux A. Springer; 2007:59-87.

13. Luengo JM, Garcia JL, Olivera EL: The phenylacetyl-CoA catabolon: a complex catabolic unit with broad biotechnological applications. Mol Microbiol 200I, 39: I434-I442.

14. Milani M, Leoni L, Rampioni G, Zennaro E, Ascenzi P, Bolognesi M: An active-like structure in the unphosphorylated Sty $R$ response regulator suggests a phosphorylation-dependent allosteric activation mechanism. Structure 2005, 13:1289-1297.

15. Leoni L, Ascenzi P, Bocedi A, Rampioni G, Castellini L, Zennaro E: Styrene-catabolism regulation in Pseudomonas fluorescens ST: phosphorylation of StyR induces dimerization and cooperative DNA-binding. Biochem Biophys Res Comm 2003, 303:926-93I.

16. Leoni L, Rampioni G, Di Stefano V, Zennaro E: Dual role of response regulator $S t y R$ in styrene catabolism regulation. Appl Environ Microbiol 2005, 71:54II-54I9.

17. Santos PM, Blatny JM, Di Bartolo I, Valla S, Zennaro E: Physiological analysis of expression of styrene degradation cluster in Pseudomonas fluorescens ST. Appl Environ Microbiol 2000, 6: I305- I 3 I0.

18. Shingler V: Integrated regulation in response to aromatic compounds: from signal sensing to attractive behaviour. Environ Microbiol 2003, 5: 1226-124I.

19. Cases I, de Lorenzo V: Promoters in the environment: transcriptional regulation in its natural context. Nat Rev Microbiol 2005, 3:105-118.

20. Santos PM, Leoni L, Di Bartolo I, Zennaro E: Integration host factor is essential for the optimal expression of styABCD operon in Pseudomonas fluorescens ST. Res Microbiol 2002, I 53:527-536.

21. McLeod SM, Johnson RC: Control of transcription by nucleoid proteins. Curr Opin Microbiol 200I, 4:152-159.

22. Sheridan SD, Benham CJ, Hatfield GW: Activation of gene expression by a novel DNA structural transmission mechanism that requires supercoiling-induced DNA duplex destabilization in an upstream activating sequence. J Biol Chem 1998, 273:21298-2।308.

23. Sze CC, Laurie $A D$, Shingler $V$ : In vivo and in vitro effects of integration host factor at the DmpR-regulated sigma(54)dependent Po promoter. J Bacteriol 200I, 183:2842-285 I.

24. Taylor BL, Zhulin IB: PAS domains: internal sensors of oxygen, redox potential and light. Microbiol Mol Biol Rev 1999, 63:479-506.

25. Arfin SM, Long AD, Ito ET, Tolleri L, Riehle MM, Paegle ES, Hatfield GW: Global gene expression profiling in Escherichia coli KI 2. J Biol Chem 2000, 275:29972-29984.

26. Aviv M, Giladi H, Schreiber G, Oppenheim AB, Glaser G: Expression of the genes coding for the Escherichia coli integration host factor are controlled by growth phase, rpoS, ppGpp and by autoregulation. Mol Microbiol 1994, 14:102I-1031.

27. Valls $M$, Buckle $M$, de Lorenzo $V$ : In vivo UV laser footprinting of the Pseudomonas putida sigma 54 Pu promoter reveals that integration host factor couples transcriptional activity to growth phase. J Biol Chem 200I, 277:2169-2175.

28. Lacal J, Busch A, Guazzaroni ME, Krell T, Ramos JL: The TodSTodT two-component regulatory system recognizes a wide range of effectors and works with DNA-bending proteins. Proc Natl Acad Sci USA 2006, 103:8191-8196. 
29. Lacal J, Guazzaroni ME, Busch A, Krell T, Ramos JL: Hierarchical binding of the TodT response regulator to its multiple recognition sites at the tod pathway operon promoter. J Mol Biol 2008, 376:325-337.

30. Cases I, de Lorenzo V: The black cat/white cat principle of signal integration in bacterial promoters. EMBO / 200I, 20: I- II

31. del Peso-Santos T, Bartolomé-Martín D, Fernández $C$, Alonso $S$, García JL, Díaz E, Shingler V, Perera J: Coregulation by phenylacetyl-coenzyme A-responsive PaaX integrates control of the upper and lower pathways for catabolism of styrene by Pseudomonas sp. strain Y2. J Bacteriol 2006, 188:48I2-482I.

32. del Castillo T, Ramos JL: Simultaneous catabolite repression between glucose and toluene metabolism in Pseudomonas putida is channeled through different signaling pathways. J Bacteriol 2007, I 89:6602-6610.

33. Sambrook J, Fritsch EF, Maniatis T: Molecular cloning: a laboratory manual 2nd edition. Cold Spring Harbor: Cold Spring Harbor Laboratory Press; 1989.

34. Hartmans S, Smits JP, Werf MJ van der, Volkering F, de Bont JA: Metabolism of styrene oxide and 2-phenylethanol in the styrene-degrading Xanthobacter strain I24X. Appl Environ Microbiol 1989, 55:2850-2855.

35. Horton RM, Hunt HD, Ho SN, Pullen JK, Pease LR: Engineering hybrid genes without the use of restriction enzymes: gene splicing by overlap extension. Gene 1989, 77:6I-68.

36. Rampioni G, Polticelli F, Bertani I, Righetti K, Venturi V, Zennaro E, Leoni L: The Pseudomonas quorum-sensing regulator RsaL belongs to the tetrahelical superclass of $\mathrm{H}-\mathrm{T}-\mathrm{H}$ proteins. J Bacteriol 2006, 189:1922-1930.

37. Figursky DH, Helinski DR: Replication of an origin-containing derivative of plasmid RK2 dependent on a plasmid function provided in trans. Proc Natl Acad Sci USA 1979, 76: 1648-1652.

38. Miller JH: Experiments in in molecular genetics Cold Spring Harbor: Cold Spring Harbor Laboratory Press; 1972.

39. Baggi G, Boga MM, Catelani E, Galli E, Treccani V: Styrene catabolism by a strain of Pseudomonas fluorescens. Syst Appl Microbiol 1983, 4:141-147.

40. Santos PM, Di Bartolo I, Blatny JM, Zennaro E, Valla S: New broadhost-range promoter probe vectors based on the plasmid RK2 replicon. FEMS Microbiol Lett 200I, 195:91-96.

Publish with Bio Med Central and every scientist can read your work free of charge

"BioMed Central will be the most significant development for disseminating the results of biomedical research in our lifetime. "

Sir Paul Nurse, Cancer Research UK

Your research papers will be:

- available free of charge to the entire biomedical community

- peer reviewed and published immediately upon acceptance

- cited in PubMed and archived on PubMed Central

- yours - you keep the copyright
BioMedcentral 